\title{
HEURISTIC MODEL OF SOLAR X-RAY SPECTRUM ACCORDING TO SATELLITE DATA FOR GEOPHYSICAL APPLICATIONS
}

\author{
J.A. Korsunskaja \\ Institute of Geospheres Dynamics, RAS, Moscow, Russia \\ jukor@idg.chph.ras.ru
}

\begin{abstract}
This paper presents a model and a computational algorithm for recovering the X-ray component of the solar spectrum based on GOES XL $(0.1-0.8 \mathrm{~nm}), \mathrm{XS}(0.05-0.4 \mathrm{~nm}$ or $0.05-0.3 \mathrm{~nm})$, and SDO QD (0.1-7 nm) data. The model relies on the emission spectrum of optically thin plasmas in the Mewe approximation, which is the temperature spectrum. An assumption is made about the possibility of representing the total spectrum as a superposition of Mewe spectra situated in the absorbing solar atmosphere to an optical depth equal to 1 for the energy corresponding to its temperature parameter. Thus, this
\end{abstract}

model is a version of the multi-temperature approximation. Spectrum parameters are determined from support functions, approximation expressions for which are given in Appendix.

Keywords: solar X-ray, spectrum model, satellite data.

\section{INTRODUCTION}

Electromagnetic solar radiation is a key factor creating Earth's ionosphere, which has a strong effect on radio wave propagation [Bryunelli, Namgaladze, 1988]. The main processes that determine the dynamics of the ionosphere have been studied and described, however radio-monitoring data shows events that cannot be explained in terms of well-known concepts. Such events involve, for example, disruptions of satellite navigation systems occurring in the conditions that in the context of the traditional approach correspond to the quiet ionosphere [Afraimovich et al., 2013].

One of the ways to identify causes of the said events is to design models that can more accurately describe the formation of the ionosphere and the dynamics of the solar spectrum. The possibility of implementing this approach is associated with the presence of solar radiation monitoring data. The longest dataset is from geostationary satellites GOES. The data includes measurements of the solar flux in two channels: XL $-0.1-0.8 \mathrm{~nm}$ or $\sim 1.5-12.4 \mathrm{keV}$ and $\mathrm{XS}-0.05-0.4 \mathrm{~nm}$ or $\sim 3-25 \mathrm{keV}$ (in GOES-8-GOES-12, to $0.3 \mathrm{~nm}$ ). Continuous data but with different resolution has been available since 1986; and for individual events, since 1974 [https://satdat.ngdc.noaa.gov/sem/goes/ data/].

Since 2002, the monitoring has been conducted with RHESSI. Its equipment consists of a solid-state $\gamma$ spectrometer, which provides spectra in a range from 3-6 $\mathrm{keV}$ to $\sim 7 \mathrm{MeV}$. A significant drawback with this satellite is its low orbits $(\sim 600 \mathrm{~km})$ and respective orbit time of $\sim 90 \mathrm{~min}$. This limits the time of continuous data acquisition to approximately one hour for photons with an energy of more than $\sim 10 \mathrm{keV}$, and even smaller value in the soft range due to their absorption by Earth's atmosphere. This data is available in electronic resources using HESSI and OSPEX packages [https:// hesperia.gsfc.nasa.gov/ rhessi2/].
In 2008, the low-orbit satellite Fermi was launched. It contains two main instruments: GBM - a monitor of $\gamma$ flares with the field of view of $4 \pi$ in a range from $8 \mathrm{keV}$ to $30 \mathrm{MeV}$, and LAT - a telescope in a range from $20 \mathrm{MeV}$ to $300 \mathrm{GeV}$ covering $20 \%$ of the sky, designed to study hard $\gamma$ flares of the Sun and Sun-like stars.

Since 2010 , data has been collected by the geostationary satellite SDO. Its equipment consists of two spectrometers based on optical spectrometry. Spectral data up to May 2014 corresponds to $5.8-106.2 \mathrm{~nm}$, and subsequent one, to $33-106.2 \mathrm{~nm}$. In addition, there are photodiodes for UV bands 17.5-21.1 nm (CH18), 23.4 $28.1 \mathrm{~nm}$ (CH26), 28-32.7 nm (CH30) and for X-ray $0.1-7 \mathrm{~nm}$ or $\sim 0.18-12.4 \mathrm{keV}$ (QD). The last detector consists of four photodiodes with differently directed axes, which allows us to locate a flare on the solar disk. All these rangesare identified by diffraction. This data is available on the website [http://lasp.colorado.edu/eve/ data_access/evewebdataproducts/].

As seen from the above, the dynamics of solar Xrays $(0.1-100 \mathrm{keV})$ is represented in detail by integral characteristics, but spectral measurements are made only for extra hard X-rays (EHXR) in 10-100 keV, with long time gaps. The solar X-rays play a significant role in forming the E and D layers of Earth's ionosphere, hence the need to reconstruct its spectrum from measured integral characteristics.

This problem was posed in [Nusinov, Chulankin, 1997]. The authors proposed a very simple and easy-touse method of approximate spectrum reconstruction within $0.1-10 \mathrm{~nm}$ from measurements within $0.1-0.8$ $\mathrm{nm}$ (corresponding to the GOES XL channel). This model is based on the approximation expression for the integral of the photon energy flux with a wavelength less than $\lambda$, obtained by processing satellite measurements made in 1970-1980s, taking into account a number of considerations relating to the impact of solar ra- 
diation on Earth's atmosphere. The formula presented in that paper has the form

$$
\begin{aligned}
& I_{\lambda}=C(\lambda) I_{0.1-0.8}^{D(\lambda)}, \text { where } \\
& C(\lambda)=C_{0} \exp \left(-C_{1} / \lambda^{C_{2}}\right), \\
& D(\lambda)=D_{0}+D_{1} / \lambda,
\end{aligned}
$$

$C_{0}=73.8, C_{1}=3.80, C_{2}=0.36, D_{0}=0.848, D_{1}=0.167, \lambda$ is the wavelength $(\mathrm{nm}), I_{\lambda}, I_{0.1-0.8}$ are the desired and base fluxes $\left(\mathrm{erg} \cdot \mathrm{s}^{-1} \cdot \mathrm{cm}^{-2}\right)\left(1 \mathrm{erg} \cdot \mathrm{s}^{-1} \cdot \mathrm{cm}^{-2}=10^{-3} \mathrm{~W} \cdot \mathrm{m}^{-2}\right)$. Hence, we get a clear expression for power spectral density

$$
\frac{d I_{\lambda}}{d \lambda}=\left(\frac{C_{1} C_{2}}{\lambda^{C_{2}}}-\frac{D_{1}}{\lambda} \ln \left(I_{0.1-0.8}\right)\right) \frac{I_{\lambda}}{\lambda},
$$

which can then be converted into the spectral photon flux density.

Meanwhile, the use of only one measuring channel and the range of applicability of Formulas (1), (2) make it impossible to utilize this model to study the events that are accompanied by significant changes in the indication of the XS channel, but small changes in the indication of the XL channel. These events have been analyzed in [Korsunskaja, 2015].

\section{Spectrum reconstruction from GOES measure- ments}

Enell et al. [2008] employed a method for reconstructing the hard X-ray (HXR) spectrum from GOES $\mathrm{XL}$ and XS measurements, using the results obtained by Garcia [1994]. The author has shown that these measurements can be used to reconstruct parameters of the temperature spectrum of the type

$$
F(E, T)=C_{\mathrm{ME}} E^{-1} T^{-1 / 2} \exp (-E / k T) G(E, T),
$$

which describes HXR. In (3), $F$ is the spectral flux density (photons $\left./ \mathrm{s} / \mathrm{cm}^{2} / \mathrm{keV}\right), E$ is the photon energy $(\mathrm{keV})$, $T$ is the temperature (K), $k$ is the Boltzmann constant, $G$ is the slowly varying dimensionless function, $C_{\mathrm{ME}}$ is the dimensional factor or emission measure, which depends on the number of electrons involved in the generation of photons and independent of $E$ and $T$. The idea of this approach is that the ratio of energy fluxes in the XL and XS channels

$$
R_{\mathrm{XR}}(T)=\int_{\mathrm{XL}} F(E, T) E d E / \int_{\mathrm{XS}} F(E, T) E d E
$$

is the monotonic function of $T$, and hence we can determine the inverse function $T_{\mathrm{XR}}(R)$. This allows us to compute $T=T_{\mathrm{XR}}(R)$, where $R=W_{\mathrm{XL}} / W_{\mathrm{XS}}$, from measured $W_{\mathrm{XL}}$ and $W_{\mathrm{XS}}$, and then to get $C_{\mathrm{ME}}$.

Enell et al. [2008] used the spectrum proposed in [Culhane, Acton, 1970]

$$
\begin{aligned}
& F(E, T)= \\
& =C \cdot E^{-1.3} T^{-0.2} \exp \left(-\frac{E}{k T}\right)\left(1-\left(\frac{E}{88.0}\right) 0.33 k T\right)^{-1}
\end{aligned}
$$

as a result of processing of rocket and satellite experimental data obtained in 1950-1960s. The range of applicability of (5) is designated by the authors as $E=1.5-15$
$\mathrm{keV}$ and $T=4-20 \mathrm{MK}$. Dimension of $C$ is not equal to dimension of $C_{\mathrm{ME}}$ in (3).

This approach has been implemented at IDG RAS by Strelkov when developing a model for calculating the electron-ion rate in the $\mathrm{D}$ and $\mathrm{E}$ layers of the ionosphere during solar flares [Lyakhov et al., 2015]. The model as part of a more general model has been tested on the experimental base of ISTP SB RAS in an HF band and received a good rating [Ponomarchuk et al., 2015]. Signal verification of the VLF band gave, however, poor results, thus making us return to the spectrum model.

To refine the model, we use the spectrum shown in [Mewe et al., 1986]. This work is the final in a series of six articles which address the problem of electron emission spectrum with Maxwell distribution of energy in optically thin plasmas in a nonrelativistic approximation. The problem reduces to deriving the function $G$ from (3). In addition to the detailed description of this function, the article presents a simplified model for calculating the continuous spectrum component and its software code in FORTRAN. We have used this very software in our work. The range of applicability of the model is defined by the authors as $\lambda=1 \div 1000 \AA \AA(\sim 12.4 \mathrm{eV}$ $-12.4 \mathrm{keV}), T=0.01 \div 100 \mathrm{MK}$.

For the Mewe spectrum in view of widths of the XL and XS channels, we derive the functions

$$
\begin{aligned}
& J_{\mathrm{XL}}(T)=\int_{\mathrm{XL}} \frac{F(E, T) E}{C_{\mathrm{ME}}} d E, \\
& J_{\mathrm{XS}}(T)=\int_{\mathrm{XS}} \frac{F(E, T) E}{C_{\mathrm{ME}}} d E, \\
& R_{X L, X S}(T)=\frac{J_{\mathrm{XL}}(T)}{J_{\mathrm{XS}}(T)}
\end{aligned}
$$

and the inverse $R_{\mathrm{XL}}$ xs function $T_{\mathrm{XL}, \mathrm{XS}}(R)$. The spectral photon flux density is determined from (3), where $T=T_{\mathrm{XL}, \mathrm{XS}}\left(W_{\mathrm{XL}} / W_{\mathrm{XS}}\right), C_{\mathrm{ME}}=W_{\mathrm{XL}} / J_{\mathrm{XL}}(\mathrm{T}), W_{\mathrm{XL}}$ and $W_{\mathrm{XS}}$ are GOES measurements.

Figure 1 compares the results of spectra reconstruction with application of the Mewe and Culhane approximations, obtained from real satellite measurements on February 15, 2011 at 01:55 UT (both the spectra are extended beyond the formal boundaries of the range of their applicability), and also shows the spectrum measured by RHESSI. The time corresponds to the period of flare development. The X-ray flux is classified as X2.1.

Obviously, preference should be given to the Mewe approximation, which agrees well with the RHESSI spectrum in the range $7-13 \mathrm{keV}$. The question about extrapolation to larger values will be discussed below. It should also be added that the spectrum in the Mewe approximation is one of the two spectra discussed in [Garcia, 1994].

Because the Mewe approximation is applicable to the entire region of interest below $10 \mathrm{keV}$, the question arises about adequacy of the constructed spectrum. To answer it, we implemented the following approach. Similarly to $J_{\mathrm{XL}}$ and $R_{\mathrm{XL}, \mathrm{XS}}$, we derived $J_{\mathrm{QD}}(T)$ and $R_{\mathrm{QD}}, \mathrm{XL}(T)$ corresponding to the SDO QD channel. It is obvious that we can speak of the adequacy when $W_{\mathrm{XL}} \cdot R_{\mathrm{QD}, \mathrm{XL}}(T)$, where 
$T=T_{\mathrm{XL}, \mathrm{XS}}\left(W_{\mathrm{XL}} / W_{\mathrm{XS}}\right)$, is close to those actually measured by SDO.

This approach was applied to the GOES and SDO data obtained on September 06, 2017 during strong solar flares and directly before them. The analysis is based on data with an increment of 1 min. Figure 2 shows satellite measurements for the three channels of interest and estimated value of the reconstructed spectrum in a range $0.1-7 \mathrm{~nm}$, which was calculated for each time interval. We can clearly see that this curve is significantly below the $W_{\mathrm{QD}}$ curve and there is no similarity between them.

The function is also plotted which is obtained by applying Formula (1) to the measured values of $W_{\mathrm{XL}}$ to calculate the integral flux in the range $0.1-7 \mathrm{~nm}$. We can see that this estimation also strongly (5-20 times) underestimates the flux, and hence Formula (2) cannot correctly describe the spectrum in soft X-rays.

\section{Spectrum reconstruction from GOES and SDO simultaneous measurements}

The spectrum of the form of (3) is the basis for describing the X-ray spectrum. This allowed us to apply the approach used above for the XS and XL channels to the XL and QD pair. For this purpose, from $R_{\mathrm{QD}, \mathrm{XL}}(T)$ we derived $T_{\mathrm{QD}, \mathrm{XL}}(R)$. The spectrum parameters are defined in this case as $T=T_{\mathrm{QD}, \mathrm{XL}}\left(W_{\mathrm{QD}} / W_{\mathrm{XL}}\right), C_{\mathrm{ME}}=$ $=R_{\mathrm{QD}, \mathrm{XL}}(T) \cdot W_{\mathrm{XL}} / J_{\mathrm{XL}}(T)$. Let us designate this spectrum $F_{\mathrm{QD}, \mathrm{XL}}(E)$; and the previously obtained one, $F_{\mathrm{XL}, \mathrm{XS}}(E)$.

Figure 3 shows the $F_{\mathrm{QD}, \mathrm{XL}}(E)$ and $F_{\mathrm{XL}, \mathrm{XS}}(E)$ spectra obtained from real satellite measurements on September 06, 2017 at 07:15 (quiet conditions) and 12:01 UT (during the flare). The temperatures $T_{\mathrm{XL}, \mathrm{XS}}$ and $T_{\mathrm{QD}, \mathrm{XL}}$ for the former are 5.03 and 2.19 respectively; for the latter, 20.79 and $2.93 \mathrm{MK}$, i.e. in both the cases they differ significantly. In addition to the calculated spectra, as a reference the LASP Reference Spectrum is shown (No. 1, March 25-29, 2008) [http://lasp.colorado.edu/ lisird/whi_ref_spectra/whi_ref_spectra.html].

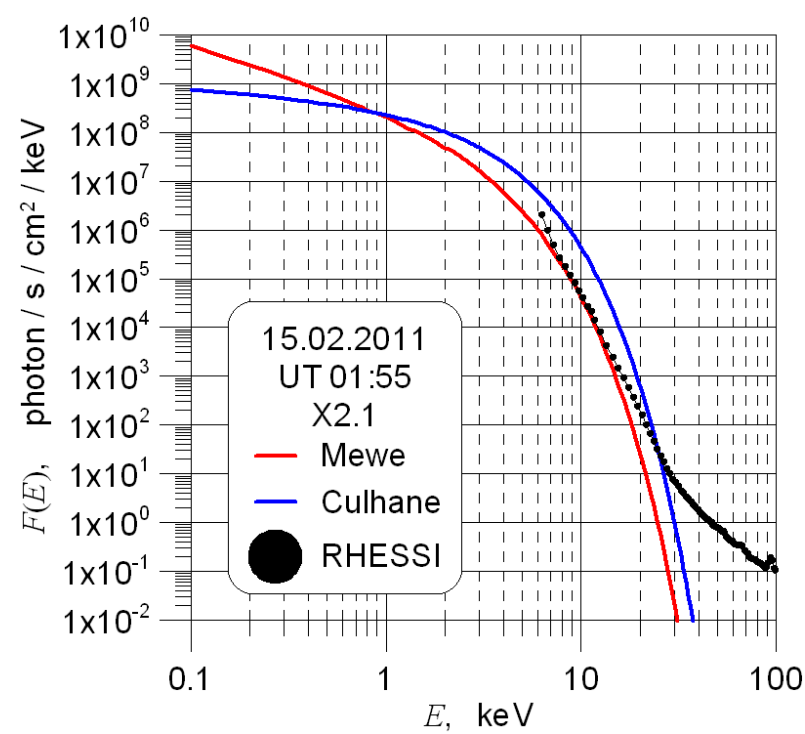

Figure 1. Spectra reconstructed using the Mewe and Culhane approximations, and RHESSI spectrum

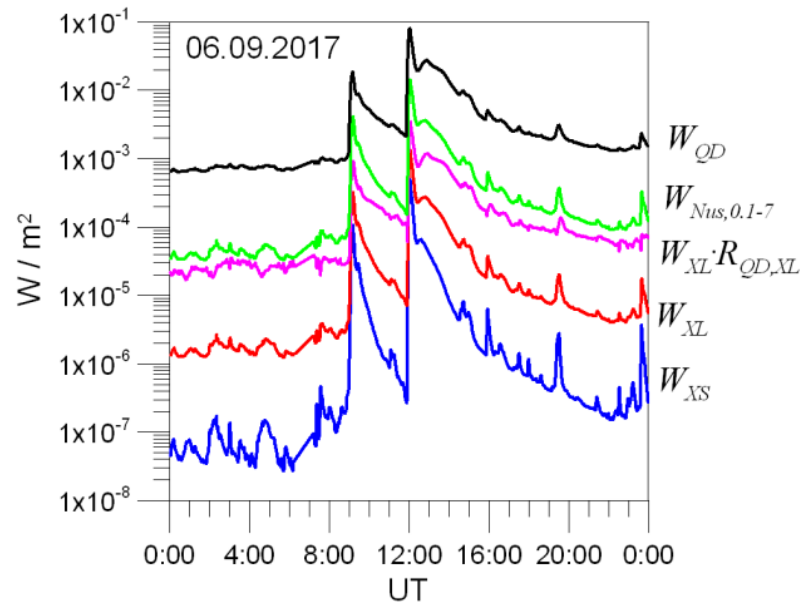

Figure 2. Measurements of fluxes in the GOES XS and $\mathrm{XL}$ channels and SDO QD channel; $W_{\mathrm{XL}} \cdot R_{\mathrm{QD}, \mathrm{XL}}$ determined from $W_{\mathrm{XS}}$ and $W_{\mathrm{XL}}$, as well as $W_{\mathrm{Nus}, 0.1-7}$ - the estimated flux in the range $0.1-7 \mathrm{~nm}$ derived from $W_{\mathrm{XL}}$ according to (1)

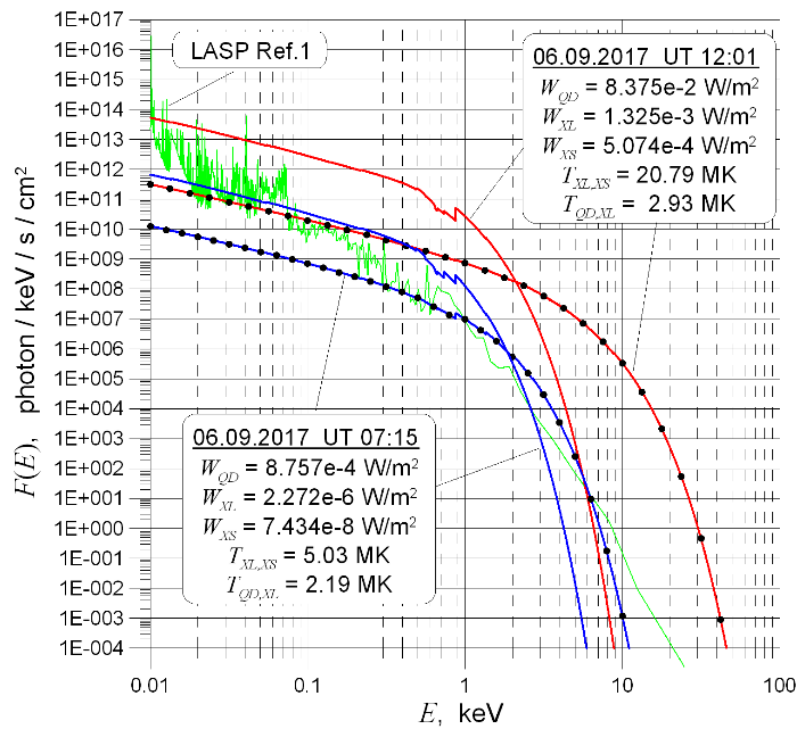

Figure 3. Spectra $F_{\mathrm{QD}, \mathrm{XL}}(E)$ (solid lines) and $F_{\mathrm{XL}, \mathrm{XS}}(E)$ (line with $\bullet$ ) calculated from actually measured values for quiet conditions (07:15 UT) and during the flare (12:01 UT), and the LASP Ref.1 spectrum

As mentioned above, the Mewe approximation is applicable not only to X-ray, but also to the UV range, shown in Figure 3. This implies that the energy flux in the UV channels at 12:01 UT should be nearly 100 times greater than their values at 07:15 UT.

However, as seen in Figure 4, the ratio of measurements in the CH18 channel $(58.7-70.7 \mathrm{eV})$ for the day considered is $\sim 1.6$ (except for the time when the satellite was in Earth's shadow); and as compared to the flux in it corresponding to the LASP spectrum, no more than 2.5. The direct calculation of energy flux in the $\mathrm{CH} 18$ channel for $F_{\mathrm{QD}, \mathrm{XL}}$ yields $8.2 \cdot 10^{-5} \mathrm{~W} / \mathrm{m}^{2}$ for $07: 15 \mathrm{UT}$ and $5.9 \cdot 10^{-3} \mathrm{~W} / \mathrm{m}^{2}$ for $12: 01 \mathrm{UT}$. If the former, which is 6 times less than the measured one, could be attributed to the use of the simplified spectrum model, the 12-fold excess of the latter over the measured ones implies a mismatch between the model and the real phenomenon. 


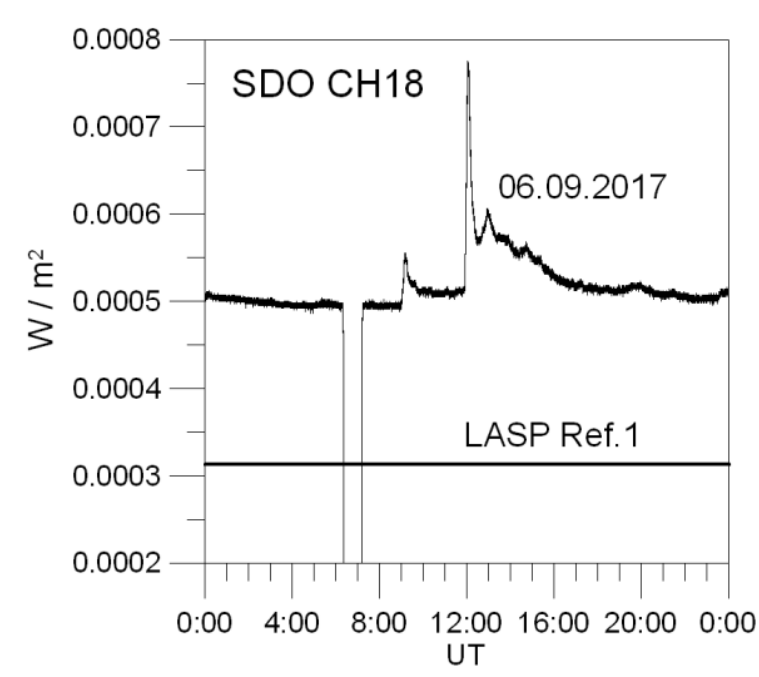

Figure 4. SDO CH18 measurements for September 06, 2017 and associated value according to the LASP Ref.1 spectrum

The Mewe approximation in use is a thin layer approximation, i.e. it describes the spectrum provided that photons are not absorbed by the medium from which they originate. This condition holds for the emission that is generated in the solar corona. Solar flares are, however, traditionally associated with sunspots, i.e. with processes developing in the lowest layer of the solar atmosphere - the photosphere. This fact suggests that the generation region is in the absorption layer but at such a depth from which the hard spectral component goes without losses, whereas the soft component is partially or fully absorbed by the medium. In this case, the absorption in the solar atmosphere occurs due to photoionization interaction with medium components, and resulting photoelectrons produce a softer spectrum, located in higher layers. This indicates that the measured spectrum can be represented as a sum of spectra from several sources.

Using this concept and making an additional a priori assumption that the depth at which the spectrum corresponding to the temperature $T$ is generated fits the optical thickness $\tau(k T)=1$, we examine the spectrum

$$
F^{*}(E, T)=F(E, T) \exp (-\sigma(E) / \sigma(k T)),
$$

where $\sigma(E)$ is the average cross-section of absorption of photons with energy $E$ in the photosphere.

In calculating the cross-section, we assume that the medium consists of neutral atoms in the ratio of the number of particles per unit volume (\%) [Eddy, 1979]: $\mathrm{H}$ 92.0949, $\mathrm{He}-7,788, \mathrm{O}-0.06033, \mathrm{C}-0.0303, \mathrm{~N}-$ $0.00806, \mathrm{Ne}-0.00752, \mathrm{Fe}-0.00358, \mathrm{Si}-0.00313, \mathrm{Mg}$ $-0.00261, \mathrm{~S}--0.00157$. The atomic particle crosssections are adapted from the EPDL97 model [https://www-nds.iaea.org/epd197/libsall.htm]. Figure 5 plots $\sigma(E)$ for the energy range from $50 \mathrm{eV}$ to $200 \mathrm{keV}$. We can see jumps corresponding to ionization thresholds of electron shells, the main of which are K-shells of oxygen, silicon, and iron. Between these energies, the function is considered continuous.

In view of the available data, the model spectrum has the form

$$
F_{\mathrm{Sol}}(E)=F_{\mathrm{XUV}}(E)+F_{\mathrm{SXR}}(E)+F_{\mathrm{HXR}}(E) .
$$

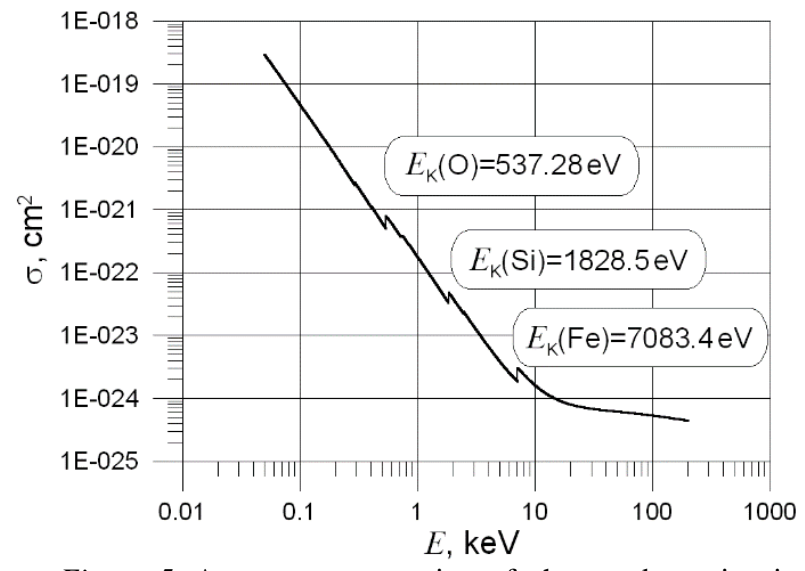

Figure 5. Average cross-section of photon absorption in the solar photosphere. Ionization thresholds of K-shells of oxygen, silicon, and iron

Suppose in this case that $F_{\mathrm{SXR}}$ and $F_{\mathrm{HXR}}$ are associated with the absorption in the solar atmosphere, whereas $F_{\text {XUV }}$ corresponds to the thin layer approximation. Each of them is characterized by its own temperature and emission measure, which are designated $T_{\mathrm{XUV}}, T_{\mathrm{SXR}}$, $T_{\mathrm{HXR}}$ and $C_{\mathrm{XUV}}, C_{\mathrm{SXR}}, C_{\mathrm{HXR}}$ respectively.

The $F_{\mathrm{HXR}}$ spectrum describes the hard component. The above considerations suggest that the other two components of spectrum (7) do not contribute to $W_{\mathrm{XS}}$, therefore its temperature is set to $T_{\mathrm{HXR}}=T_{\mathrm{XL}, \mathrm{XS}}\left(W_{\mathrm{XL}} / W_{\mathrm{XS}}\right)$.

Obviously, the presence of absorption changes the contribution of the source to the emission spectrum, which is determined by the functions $J^{*}{ }_{\mathrm{Xs}}(T), J_{\mathrm{XL}}^{*}(T)$, $J^{*}{ }_{\mathrm{QD}}(T)$. These functions are defined for spectrum (6) similarly to those without ${ }^{*}$. Hence, to ensure the required value in the XS channel the emission measure for $F_{\mathrm{HXR}}$ should be increased $1 / K\left(T_{\mathrm{HXR}}\right)$ times as compared to $F_{\mathrm{XL}, \mathrm{XS}}$, where $K(T)=J_{\mathrm{XS}}^{*}(T) / J_{\mathrm{XS}}(T)$. Then $C_{\mathrm{HXR}}=W_{\mathrm{XL}} / J_{\mathrm{XL}}\left(T_{\mathrm{HXR}}\right) / K\left(T_{\mathrm{HXR}}\right)$.

The contribution of the thus constructed spectrum in the XL channel is $W_{\mathrm{XL}}, \mathrm{HXR}=C_{\mathrm{HXR}} \cdot J^{*}{ }_{\mathrm{XL}}\left(T_{\mathrm{HXR}}\right)$; and in the QD channel, $W_{\mathrm{QD}, \mathrm{HXR}}=W_{\mathrm{XL}, \mathrm{HXR}} \cdot R_{\mathrm{QD}, \mathrm{XL}}^{*}\left(T_{\mathrm{HXR}}\right)$, where $R_{\mathrm{QD}, \mathrm{XL}}^{*}(T)=J_{\mathrm{QD}}^{*}(T) / J_{\mathrm{XL}}^{*}(T)$.

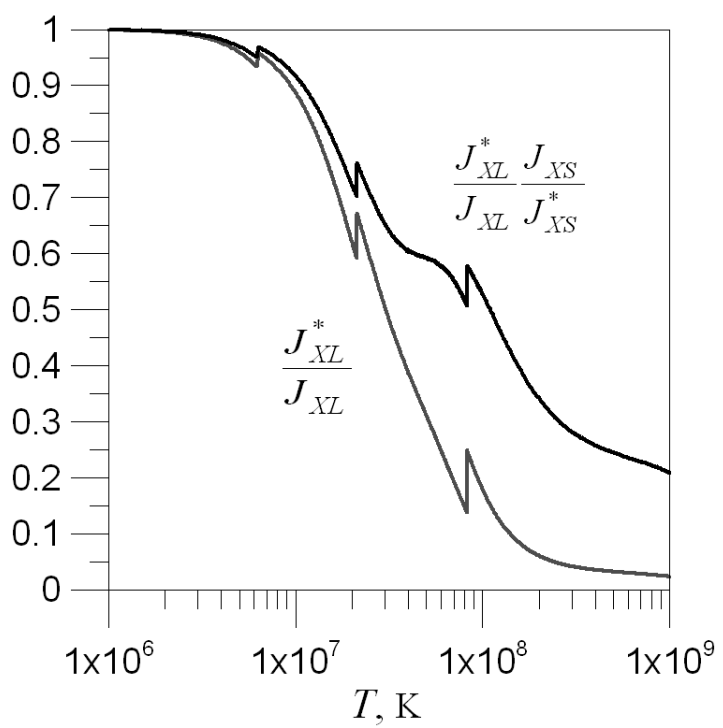

Figure 6. Contribution of $W_{\mathrm{XL}, \mathrm{HXR}}$ to $W_{\mathrm{XL}}$ with and without regard to the correction related to the XS channel 
Note that with increasing temperature the contribution of the correction associated with the XS channel increases. Figure 6 plots the contribution of $W_{\mathrm{XL}, \mathrm{HXR}}$ to $W_{\mathrm{XL}}$ with and without regard to the correction.

The remaining fluxes in the QD and XL channels should be divided between $F_{\mathrm{SXR}}$ and $F_{\mathrm{XUV}}$. Available data on measurements in the X-ray band is inadequate to provide parameters of both the spectra. Fluxes in the $\mathrm{CH} 18, \mathrm{CH} 26$, and $\mathrm{CH} 30$ channels of the UV band are determined by recombination processes of FeIX and HeII (two channels) [Woods et al., 2012] and refer to the part of the spectrum which is not described by the temperature approximation in use. We therefore assume that the spectrum $F_{\mathrm{XUV}}$ contributes $W_{0}=2 \cdot 10^{-5} \mathrm{~W} / \mathrm{m}^{2}$ to the QD channel, and the spectrum temperature $T_{\mathrm{XUV}}=0.7$ MK. The emission measure is defined as $C_{\mathrm{XUV}}=W_{0}$ $/ J_{\mathrm{QD}}\left(T_{\mathrm{XUV}}\right)=W_{0} / R_{\mathrm{QD}, \mathrm{XL}}\left(T_{\mathrm{XUV}}\right) / J_{\mathrm{XL}}\left(T_{\mathrm{XUV}}\right)$. The value of $W_{0} / R_{\mathrm{QD}, \mathrm{XL}}\left(T_{\mathrm{XUV}}\right)$ determines the contribution of this spectrum to the XL channel.

By way of illustration, Figure 7 shows all the spectra of interest based on data corresponding to the above event of September 06, 2017, 12:01 UT. The Figure also shows the LASP Ref. 1 spectrum. It is evident that $F_{\mathrm{XUV}}$ is a "pedestal" on which a part of the LASP Ref. 1 spectrum rests for $E<500 \mathrm{eV}$. The selected value of $W_{0}$ does not contradict the measuring results. The value of $W_{\mathrm{QD}}$ corresponding to the LASP Ref. 1 spectrum is $1.471 \cdot 10^{-4}$ $\mathrm{W} / \mathrm{m}^{2}$, and the minimum daily average value recorded in SDO for the entire observation period through June 2018 , is not lower than $6 \cdot 10^{-5} \mathrm{~W} / \mathrm{m}^{2}$.

Thus, $F_{\text {SXR }}$ should be built on the assumption that its contributions to the QD and XL channels are $W_{\mathrm{QD}, \mathrm{SXR}}=W_{\mathrm{QD}}-W_{\mathrm{QD}, \mathrm{HXR}}-W_{0}$ and $W_{\mathrm{XL}, \mathrm{SXR}}=W_{\mathrm{XL}}-W_{\mathrm{XL}, \mathrm{HXR}}$ respectively. It is not necessary to make a correction for the XL channel with respect to $F_{\mathrm{XUV}}$ since the formal computation of this value yields $1.25 \cdot 10^{-14} \mathrm{~W} / \mathrm{m}^{2}$, which is at least 5 orders of magnitude lower than the lower limit of measurements. To determine temperature, we constructed an additional function $T_{\mathrm{QD}, \mathrm{XL}}^{*}(R)$ inverse to $R_{\mathrm{QD}, \mathrm{XL}}^{*}$. Parameters of $F_{\mathrm{SXR}}$ are $T_{\mathrm{SXR}}=T^{*} \mathrm{QD}, \mathrm{XL}\left(W_{\mathrm{QD}, \mathrm{SXR}} / W_{\mathrm{XL}}\right.$, SXR $), C_{\mathrm{SXR}}=W_{\mathrm{XL}, \mathrm{SXR}} / J_{\mathrm{XL}}^{*}\left(T_{\mathrm{SXR}}\right)$.

Figure 8 shows diurnal variations of $T_{\mathrm{QD}, \mathrm{XL}}, T_{\mathrm{SXR}}$, and $T_{\mathrm{HXR}}$, calculated from September 06, 2017 measurements. We can clearly see that throughout the period $T_{\mathrm{SXR}}$ is lower than $T_{\mathrm{QD}, \mathrm{XL}}$. Note also that if the range of variations in $T_{\mathrm{SXR}}$ was less than 1.1 MK, $T_{\mathrm{HXR}}$ varied within $19 \mathrm{MK}$.

\section{Behavior of spectrum in a high-energy range}

As follows from (3) and Figure 7, the temperature spectrum at $E \gg k T$ decreases exponentially. However, as noted in the review by Dennis [1988], the temperature spectrum at high energies becomes power, i.e. $\sim E^{-\gamma}$. The value $\gamma$ varies quite widely and depends on the type of flare and its phase. For many flares $\gamma$ is within 7-9, but in some cases it falls to $\sim 5$ and below. In this case, referring to Figure 1, when leaving for a harder region the spectrum, remaining power, is described by a function with smaller $\gamma$.

Thus, if it is necessary to study in detail the effect of hard ultra-high temperature solar emission component on Earth's atmosphere, we need spectrometer data. At

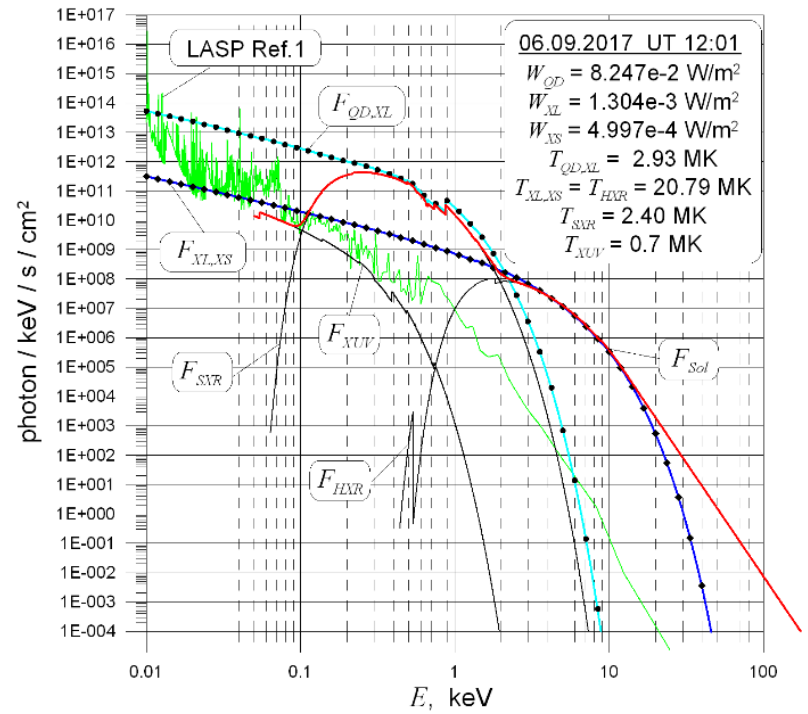

Figure 7. Example of spectrum construction from $W_{X S}$, $W_{X L}$, and $W_{Q D}$. The $F_{\text {Sol }}$ spectrum is constructed using an extrapolation algorithm

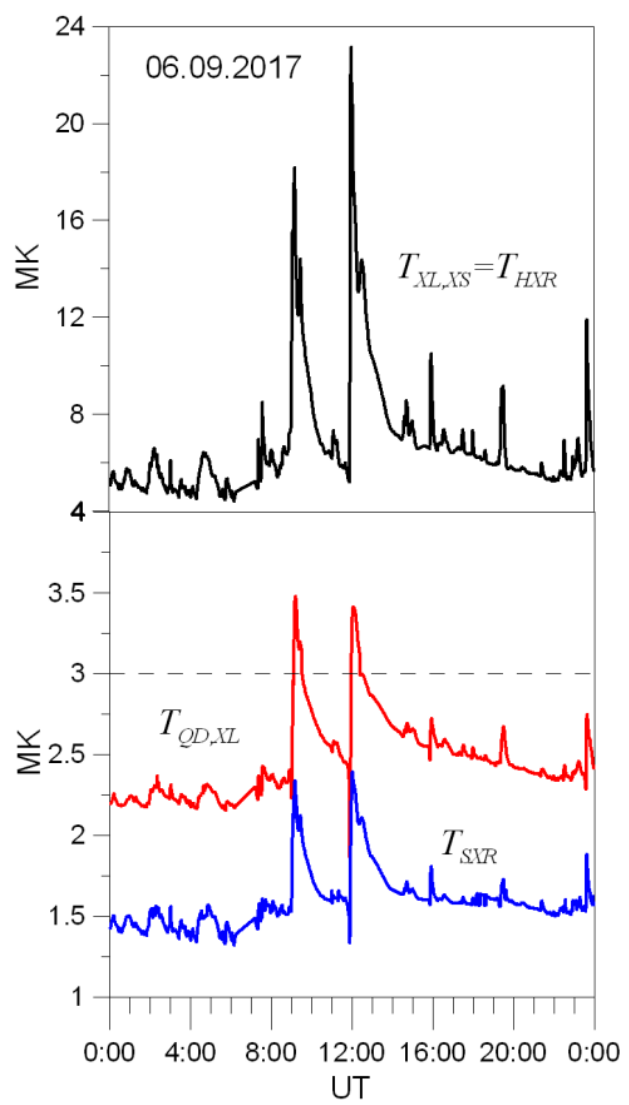

Figure 8. Temperatures measured on September 06, 2017

the same time, their analysis has shown that in a first approximation we may restrict ourselves to extrapolation of spectrum (7)

$$
F_{\text {Sol }}(E)=F_{\text {Sol }}\left(E_{0}\right) \cdot\left(E / E_{0}\right)^{-\gamma} \text {, }
$$

where $E_{0}=6.5 k T_{\mathrm{HXR}}, \gamma=-\left.\frac{d \ln F_{\mathrm{HXR}}}{d \ln E}\right|_{E=E_{0}}, E>E_{0}$. Such an approximation yields $\gamma \approx 7.6 \pm 0.2$. The $F_{\text {Sol }}$ spectrum shown in Figure 7 demonstrates this extrapolation method. 
The $F_{\text {Sol }}$ spectrum does not always provide a smooth conjugation with experimentally derived spectra. To build a single experimental model spectrum, which contains no physically unreasonable jumps, we can adopt the following modification of the above method.

From $W_{\mathrm{XL}}$ and experimental spectrum given by a set of points $\left\{\left(E_{\text {data }, i}, F_{\text {data }, i}\right) \mid i=1, N_{\text {data }}\right\}$, find $T_{\text {ex }}$ such that $F(E$, $\left.T_{\mathrm{ex}}\right)$ with $C_{\mathrm{ME}}=W_{\mathrm{XL}} / J_{\mathrm{XL}}\left(T_{\mathrm{ex}}\right)$ is sufficiently close to $F_{\text {data }}$. The formal criterion in this case takes the form

$$
\left|\max _{i}\left(\frac{F\left(E_{\mathrm{data}, i}, T_{\mathrm{ex}}\right)}{F_{\text {data }, i}}-1\right)\right| \leq \varepsilon,
$$

where $\varepsilon$ is the preset value. Assume further that $T_{\mathrm{HXR}} \equiv T_{\mathrm{ex}}$ and the model part of the spectrum is constructed in accordance with the procedure described above. Obviously, extrapolation (8) in this case is not made.

\section{Features of the computational algorithm and ex- perimental data}

We have developed a software implementation of the proposed model. The developed algorithm is briefly outlined in Appendix 1. The reference functions used to compute spectrum parameters are calculated by the approximations. Their coefficients are given in Appendix 2. The approximation coefficients are given with a precision of no more than six mantissa digits.

Obviously, ideally the integrals $\int F_{\text {Sol }}(E) E d E$, calculated from ranges corresponding to the $\mathrm{XS}, \mathrm{XL}$, and QD channels should give the initial values of energy fluxes in them. The calculations have revealed the following relative errors: $\pm 1 \%$ for $\mathrm{XL}$, from -2 to $3 \%$ for $\mathrm{XS}$, from +1 to $+5 \%$ for QD. These errors are mainly related to the quality of the approximation expressions in use.

Note that these values correspond to the spectrum without extrapolation. Extrapolation has an effect only on the XS error, which in this case may be as great as several hundred percent. This situation is observed when the extrapolated part of the spectrum makes a significant contribution to the integral and only for the quiet Sun.

In general, we can argue that the higher is spectrum rigidity and the greater are the fluxes, the higher is the calculation accuracy.

It is important to note another feature of the developed model. The $G(E, T)$ function in use does not have monotonicity and continuity properties in any of the variables, thereby $R_{\mathrm{XL}, \mathrm{XS}}(T), R_{\mathrm{QD}, \mathrm{XL}}(T)$, and $R_{\mathrm{QD}, \mathrm{XL}}^{*}(T)$ do not have these properties either. In the range that is of practical importance for the problem considered, singular points are temperatures of $1,1.5,3,4.5$, and 8 MK. A noticeable effect occurs, however, only when $T=3 \mathrm{MK}$, which manifests itself in the inability to define $T_{\mathrm{XL}, \mathrm{XS}}(R)$ and $T_{\mathrm{QD}, \mathrm{XL}}^{*}(R)$ in the vicinity of this point as inverse and in the need to complete them through additional considerations. At the same time, the temperature of the hard spectral component of $\sim 3 \mathrm{MK}$ corresponds to the quiet Sun, i.e. to the situation when the spectrum is largely determined by the extrapolation model adopted.
For the soft component, this value is too high. It was reached in none of the cases considered. Thus, a certain voluntarism in determining these functions do not affect the final result.

The difference of this realization from the described theoretical model is that, as experience has shown, the consideration of the correction $J_{\mathrm{XS}}^{*} / J_{\mathrm{XS}}$ at low $T_{\mathrm{HXR}}$ yields a greater error of the energy flux integral as compared to the version when this correction is not made. It was therefore decided to take $K(T)=1$ for $k T<E_{\mathrm{K}}(\mathrm{O})$, i.e. the contribution of $W_{\mathrm{XL}, \mathrm{HXR}}$ to $W_{\mathrm{XL}}$ (Figure 6) for temperatures to the left of the left jump corresponds to the lower curve, not to the upper one.

As stated above, GOES experimental data corresponds to two determinations of the XS range (0.05-0.4 and $0.05-0.3 \mathrm{~nm})$, so the support functions $T_{\mathrm{XL}, \mathrm{XS}}(R)$ and $K(T)$ dependent on the channel width are presented in Appendix 2 for both the determinations. The main volume of SDO experimental data falls, however, in the period of operation of GOES satellites with equipment corresponding to $0.05-0.4 \mathrm{~nm}$, so all the results reported in this paper relate to this case.

It is also important to remember that according to [GOES_XRS_readme], to obtain real values of fluxes the archival data from satellites with numbers 8-15 should be rescaled by dividing the read value of the XL channel by 0.7 ; and of the XS channel, by 0.85 .

In most cases, at the same time two or more GOES satellites worked. This made it possible to obtain a continuous series of experimental data even when one of the satellites was in Earth's shadow or was shut down for maintenance. However, simultaneous measurements made by different satellites differ. The greatest differences are observed at low solar activity. Data analysis has shown that as basic measurements we should take those from GOES-15. The lower limit of archival values from $\mathrm{XS}_{13}$ (subscript is the satellite number) is $2 \cdot 10^{-8} \mathrm{~W} / \mathrm{m}^{2}$, therefore there is a 20 -fold difference from $\mathrm{XS}_{15}$. It is not recommended to use the $\mathrm{XL}_{14}$ and $\mathrm{XS}_{14}$ values if they are below $10^{-7} \mathrm{~W} / \mathrm{m}^{2}$. The data from satellites $8-12$ was not analyzed.

When using satellite data, we should remember that GOES measurements are assigned to Earth's orbit, whereas almost all SDO material and all RHESSI and Fermi data are assigned to $1 \mathrm{AU}$. Daily average factors of conversion to $1 \mathrm{AU}$ (squared distance from the Sun to Earth in AU) are held in the GOES archive. Its associated correction is not, however, beyond $\pm 3.4 \%$, therefore its consideration can be deemed excessive for geophysics.

When constructing the experimental model spectrum, it does not make sense to consider experimental points farther than $\sim 30 \mathrm{keV}$, and we should restrict ourselves to the part that corresponds to the domain of monotonous decrease.

\section{RESULTS AND DISCUSSION}

To assess the quality of the proposed model, we have compared the model spectra $F_{\text {Sol }}$ derived from actual measurements with RHESSI spectra, as well as with the model spectra $F_{\text {Nus }}$, calculated by Formulas (1), (2) for the same initial data, in a photon energy range $0.124-12.4 \mathrm{keV}$. 

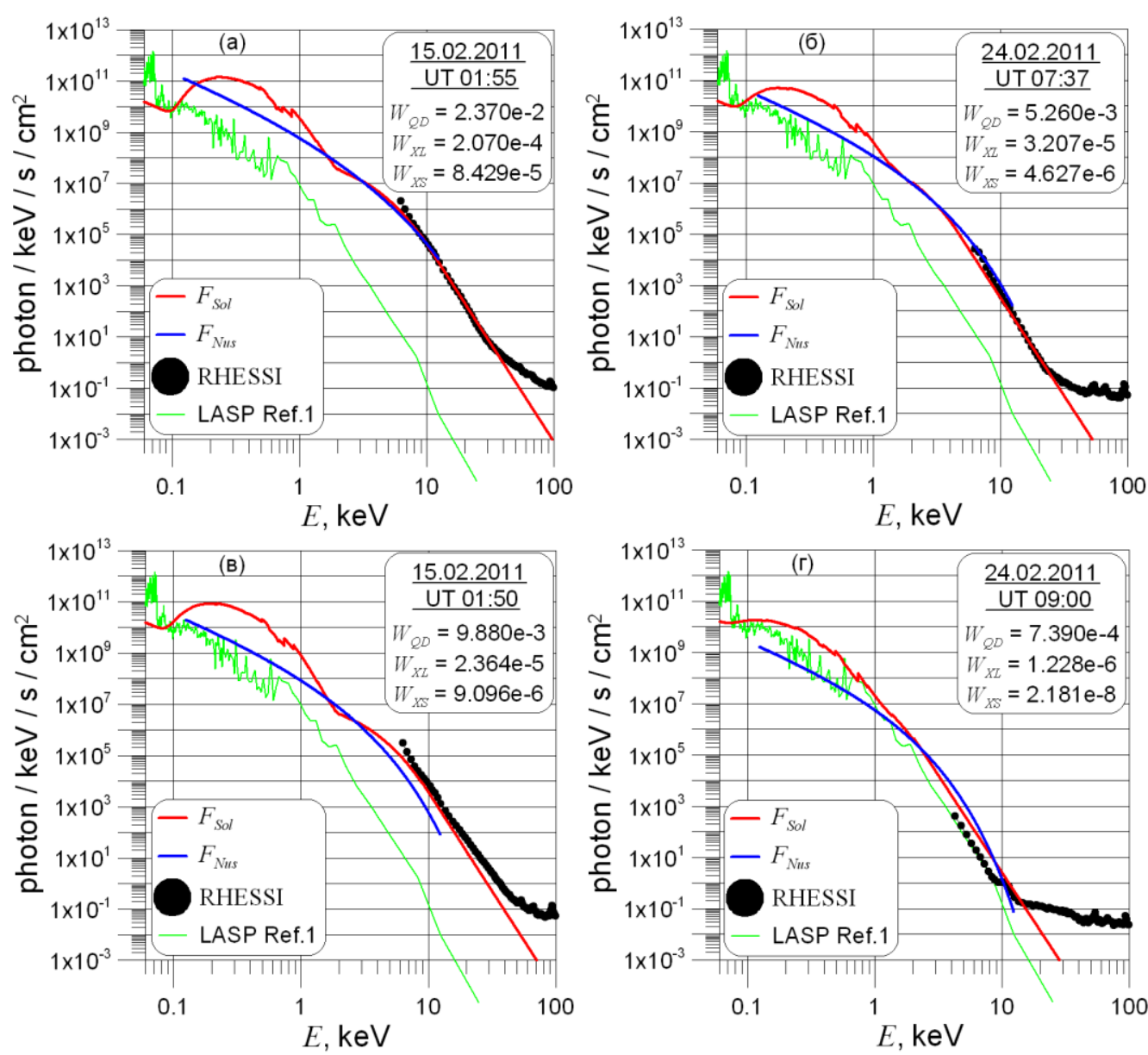

Figure 9. Comparison of the model spectra $F_{\text {Sol }}$ and $F_{\text {Nus }}$ with experimental data

Figure 9 shows spectra corresponding to X2.1, M3.2, M2.4, and C1.2 fluxes. We can see that in the first two cases, the $F_{\text {Sol }}$ spectrum agrees well with the RHESSI spectrum. The model spectra for energies higher than $\sim 1.5 \mathrm{keV}$ coincide or are very close.

In the situation illustrated in Figure 9, $c$, the model spectrum is slightly lower than the experimental one, and extrapolation yields a greater rate of decrease as compared to the real one. The reason may lie in the fact that, as noted in [Dennis et al., 2006], in some cases the low-energy part of the RHESSI spectrum corresponds to a multi-temperature approximation. Obviously, in this situation approximation (7) in use is not sufficient for a correct description of the spectrum. The model spectrum $F_{\text {Nus }}$ is located still lower than $F_{\text {Sol }}$.

The $F_{\text {Sol }}$ spectrum (Figure $9, d$ ) in the HXR range is located slightly higher than the RHESSI spectrum, but its decrease rate is close to experimental values. It is also close to the hard part of $F_{\text {Nus }}$, but better describes the decrease.

Analysis of the spectra shows that the lower is the flux class, the worse is the agreement between model and experimental spectra. This is probably due to GOES equipment characteristics and the model of conversion of measuring results to energy fluxes. Widths of the $\mathrm{XL}$ and XS channels designated as $0.1-0.8$ and $0.05-0.4 \mathrm{~nm}$, are only particular effective values. Real transmission windows calculated for GOES-13 and presented in
[GOES_XRS_readme] are defined as 0.02-1.6 and 0.01$0.8 \mathrm{~nm}$ respectively. This means that when the temperature part of the solar spectrum is relatively small, an increase in fluxes in the EHXR range may significantly affect a measured value. An example of such a spectrum is given in Figure 10, $a$.

Figure 10, $b$ demonstrates another problem we faced with. As follows from the plotted values, fluxes in the XS, XL, and QD channels on February 16, 2018 were significantly lower than those on September 12, 2017. Behavior of the model spectra $F_{\text {Sol }}$ fully complies with them. The RHESSI spectrum on February 16, 2018 in an energy range below $\sim 20 \mathrm{keV}$ is, however, well above its spectrum for September 12, 2017. This difference may be as large as two orders of magnitude. Thus, there is a clear inconsistency between measuring results obtained by different satellites. We do not know the reason for this, but we can assume that it is associated with the problems arisen with RHESSI detectors, which were shut down because of overheating on April 11, 2018.

Referring to Figures 9 and 10, $a$, in the soft X-ray the $F_{\text {Nus }}$ spectrum is considerably lower than $F_{\text {Sol }}$. This is entirely consistent with the previously made statement that the flux value in the range equivalent to the width of the QD channel, calculated with Formula (1), is considerably less than actual measurements. Therefore, to test the proposed model we have adopted the 

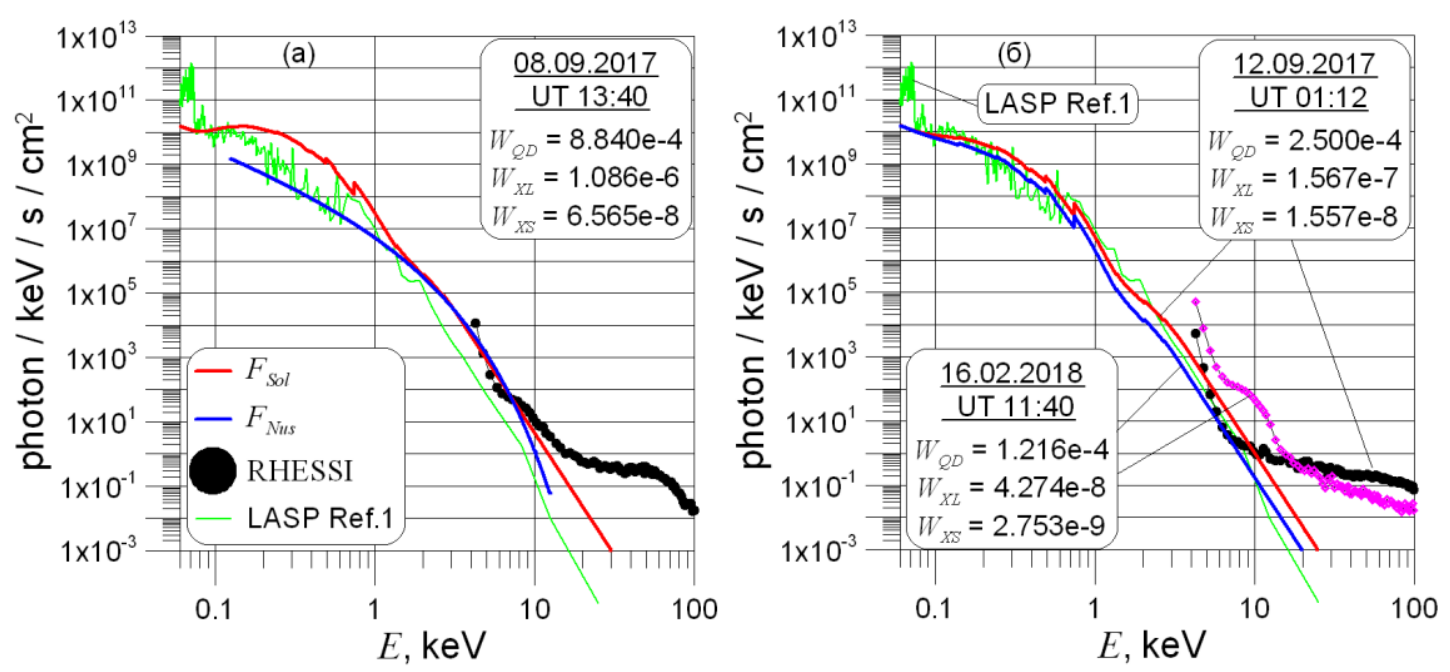

Figure 10. The same as in Figure 9 but for C1.1 at a higher flux in the EHXR range (a). Spectra $F_{\text {Sol }}$ and RHESSI for two moments of time, demonstrating a mismatch between data from different satellites (b). $W_{\mathrm{XS}}$ and $W_{\mathrm{XL}}$ are uncorrected. Units of measurements $-\mathrm{W} / \mathrm{m}^{2}$
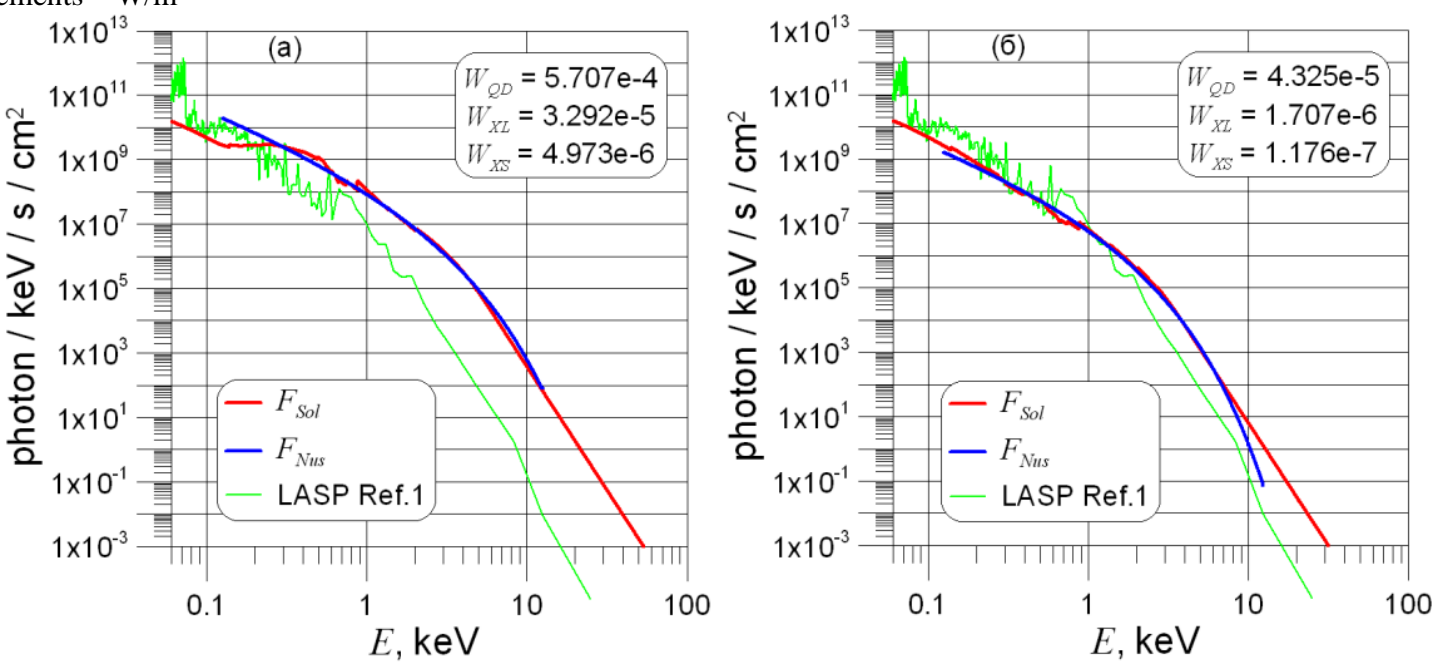

Figure 11. Comparison between $F_{\mathrm{Nus}}$ and $F_{\mathrm{Sol}}$, constructed from $W_{\mathrm{QD}}$ and $W_{\mathrm{XS}}$, calculated by Formula (1) according to $W_{\mathrm{XL}}$. Panels (a) and (b) correspond to Figure 9 , c, d. $W_{\mathrm{XL}}$ is a corrected value. Units of measurements $-\mathrm{W} / \mathrm{m}^{2}$

following approach. Given that the model provides a wide choice in setting input flux values in the three channels, we examine cases when an input parameter is $W_{\mathrm{XL}}$, and $W_{\mathrm{QD}}$ and $W_{\mathrm{XS}}$ are calculated using Formula (1).

Figure 11 shows two versions of such calculations. They correspond to the spectra in Figure $9, c, d$, for which we obtained the greatest difference between the two model spectra. The flux values $W_{\mathrm{XL}}$ plotted are corrected; and $W_{\mathrm{QD}}$ and $W_{\mathrm{XS}}$ are calculated with Formula (1). The $F_{\text {Nus }}$ and $F_{\text {Sol }}$ spectra in Figure $11, a$ in fact coincide at energies above $0.23 \mathrm{keV}$, and for the version shown in Figure 11, $b$ a significant difference takes place only at energies above $\sim 8 \mathrm{keV}$.

Concluding the discussion, let us compare the LASP Ref.1 spectrum with the model spectra $F_{\text {Nus }}$ and $F_{\text {Sol }}$, obtained from integral flux characteristics calculated directly from the reference spectrum. Their values, as well as spectra themselves, are shown in Figure 12. We can see that $F_{\text {Sol }}$ in the soft $\mathrm{X}$-ray range is closer to the reference spectrum than $F_{\text {Nus }}$, and in the range $2-8 \mathrm{keV}$ all the three spectra are nearly identical. Excess of $F_{\text {Sol }}$ over LASP Ref.1 in a harder part is of no consequence since, as seen in Figure 10, even for small fluxes in the XL and XS channels RHESSI spectra are above LASP Ref.1.

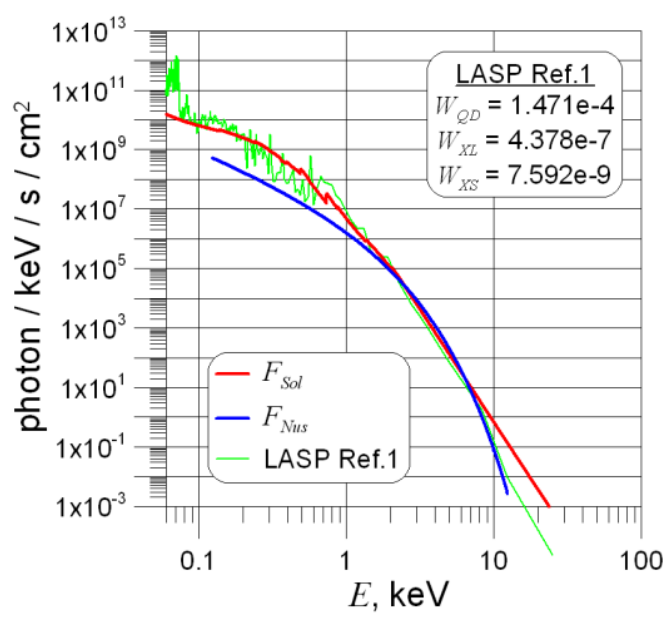

Figure 12. The LASP Ref.1 spectrum and model spectra $F_{\text {Nus }}$ and $F_{\text {Sol }}$, derived from integral characteristics computed from the reference spectrum 
The computation error depending on the accuracy in setting support functions needed to calculate parameters of the model spectrum has no significant effect on the final result.

The application of the model to real satellite data provides spectra that do not contain a significant component in the UV band, hence the lower boundary of the model spectra constructed from the complete model is the boundary of the $100 \mathrm{eV} \mathrm{X-ray} \mathrm{band.} \mathrm{In} \mathrm{the} \mathrm{absence}$ of SDO data, it is possible to build spectra for photon energies above $1.5-2 \mathrm{keV}$.

The upper limit of the application of the model spectrum is determined by the spectral photon flux density. The comparison of this spectrum with RHESSI spectra has shown that this limit may be set at $10^{-2}$ photon $/ \mathrm{keV} / \mathrm{s} / \mathrm{cm}^{2}$. The energy value corresponding to such a flux varies from 10 to $100 \mathrm{keV}$. To examine the impact of the hard solar spectrum component on Earth's atmosphere in detail, we should study the experimental model spectrum (the method of its construction is also discussed in this paper).

The presented approach for constructing spectrum from satellite measurements leaves the possibility of its refinement, one of the ways of which reduces to determination of $F_{\text {XUV }}$ parameters from real measurements in an UV band.

It should be added that the developed model is fundamentally a version of the construction of the multitemperature spectrum.

All experimental satellite data used in this study was taken from electronic resources from January to May 2018.

\section{CONCLUSION}

The paper describes the general principles of construction of X-ray solar spectrum components from GOES XS, XL and SDO QD measurements in the multi-temperature approximation based on the temperature spectrum in the Mewe approximation [Mewe et al., 1986]. The comparison of the model spectrum with RHESSI data allowed us to determine the boundary of the conversion of the temperature spectrum into the power one.

The algorithm and approximation expressions of support functions for calculating spectral parameters are given in Appendix.

During the preparation of the article, it was reported that after 16 years of operation instead of the planned two the RHESSI satellite ceased to exist as a scientific instrument.

The work was carried out under State task No. 01462019-0010.

\section{REFERENCES}

Afraimovich E, Astafyeva E, Demyanov V, Edemskiy I, Gavrilyuk N, et al. A review of GPS/GLONASS studies of the ionospheric response to natural and anthropogenic processes and phenomena. J. Space Weather and Space Climate.2013, vol. 3, no. A27. DOI: 10.1051/swsc/2013049.

BryunelliB.E., Namgaladze A.A. Fizika ionosfery [Physics of the Ionosphere]. Moscow, Nauka Publ., 1988. 528 p. (In Russian).

Culhane J.L., Acton L.W. A simplified thermal continuum function for the X-ray emission from coronal plasmas. Month ly Notices of the Royal Astronomical Society. 1970, vol. 151, pp. 141-147. DOI: $10.1093 / \mathrm{mnras} / 151.1 .141$.

Dennis B.R. Solar flare hard X-ray observations. Solar Phys. 1988, vol. 118, pp. 49-94. DOI: 10.1007/BF00148588.

Dennis B.R., Phillips K.J.H., Sylwester J., Sylwester B., Schwartz R.A., Tolbert K.A.Thermal and nonthermal contributions to the solar flare X-ray flux. 2006. URL: http://www.cbk.pan.wroc.pl/body/publikacje/2006/therm_nont h_htm.html (Accessed 21.01.2018).

Eddy J. A New Sun: The Solar Results from Skylab. National Aeronautics and Space Administration, Washington, D.C., 1979. P. 37. URL: https://history.nasa.gov/SP-402/ contents.htm.

Enell C.-F., Verronen P.T., Beharrell M.J., Vierinen J.P., Kero A., Seppala A., et al. Case study of the mesospheric and lower thermospheric effects of solar X-ray flares: coupled ionneutral modeling and comparison with EISCAT and riometer measurements. Ann. Geophys. 2008, vol. 26, pp. 2311-2321. www.ann-geophys.net/26/2311/2008/.

Garcia H.A. Temperature and emission measure from GOES soft X-ray measurements. Solar Phys. 1994, vol. 154, pp. 275-308. DOI: 10.1007/BF00681100.

GOES X-ray Sensor (XRS) Measurements. Version 1.4.1. URL: https://www.ngdc.noaa.gov/stp/satellite/goes/doc/GOES_ XRS_readme.pdf (Accessed 11.02.2018).

Korsunskaja J.A. The effect of hard X-rays and gamma radiation of the Sun on the Earth's ionosphere and other processes in the geosphere. Part I. Experimental data. Dinamicheskie protsessy $v$ geosferakh. [Dynamic Processes in Geospheres]. Moscow, GEOS Publ., 2015. pp. 122-133. (In Russian).

Lyakhov A.N. et al. Programma dlya EVM IDG-DS [IDGDS PC Program]. Patent RF no. 2015612232, 2015.

Mewe R., Lemen J.R., van den Oord G.H.J. Calculated Xradiation from optically thin plasmas. VI. Improved calculations for continuum emission and approximation formulae for nonrelativistic average Gaunt factors. Astron. Astrophys.: Suppl. Ser.1986, vol. 65, pp.511-536.

Nusinov A.A., Chulankin D.I. Variations of the solar soft X-rays during flares. Geomagnetism and Aeronomy. 1997, vol. 37, no. 1, pp. 14-23.

Ponomarchuk S.N., Kurkin V.I., Lyakhov A.N., RomanovaE.B., TashchilinA.V.The modeling of HF radio wave propagation characteristics during the periods of solar flares. Proc. SPIE 9680, 21st International Symposium Atmospheric and Ocean Optics: Atmospheric Physics. 96805F. DOI: 10.1117/12.2203591.

Woods T.N., F.G. Eparvier, Hock R., Jones A.R., Woodraska D., Judge D., et al. Extreme Ultraviolet Variability Experiment (EVE) on the Solar Dynamics Observatory (SDO): Overview of science objectives, instrument design, data products, and model developments. Solar Phys. 2012, vol. 275, pp.115-143. DOI:10.1007/s11207-009-9487-6.

How to cite this article

Korsunskaja J.A. Heuristic model of solar X-ray spectrum according to satellite data for geophysical applications. Solar-Terrestrial Physics. 2019. Vol. 5. Iss. 3. P. 75-86. DOI: 10/12737/stp-53201909.

\section{Appendix 1. Description of the HMSXS algo- rithm (Heuristic Model of Solar X-ray Spectrum)}

We present two versions of the model: complete for the $100 \mathrm{eV}$ photon energy range and short - for the $>1.5 \mathrm{keV}$ range.

The input data for the complete version is $W_{\mathrm{XL}}$ and $W_{\mathrm{XS}}$ - GOES measurements within $0.1-0.8$ and $0.05-$ $0.4 \mathrm{~nm}$ (or $0.05-0.3 \mathrm{~nm}$ ) respectively, $W_{\mathrm{QD}}$ are $\mathrm{SDO}$ measurements within $0.1-7 \mathrm{~nm}$, expressed in $\mathrm{keV} / \mathrm{s} / \mathrm{cm}^{2}$ 
$\left(1 \mathrm{~W} / \mathrm{m}^{2}=6.25 \cdot 10^{11} \mathrm{keV} / \mathrm{s} / \mathrm{cm}^{2}\right), E$ is the photon energy in $\mathrm{keV}$. The short version does not use SDO measurements.

Results of the calculations are the spectral photon flux density in photon $/ \mathrm{s} / \mathrm{cm}^{2} / \mathrm{keV}$ corresponding to $E$. The spectrum consists of two parts: temperature (at $E<E_{0}$ ) and non-temperature (at $E \geq E_{0}$ ). The value of $E_{0}$ depends on input data.

The model uses support functions: $J_{\mathrm{XL}}(T), J_{\mathrm{XL}}^{*}(T)$, $T_{\mathrm{XL}, \mathrm{XS}}(R), T_{\mathrm{QD}, \mathrm{XL}}^{*}(R), R_{\mathrm{QD}, \mathrm{XL}}(T), R_{\mathrm{QD}, \mathrm{XL}}^{*}(T), K(T), \sigma(E)$; approximation expressions for their calculations are given in Appendix 2. Arguments and functions of $T$ are expressed in $\mathrm{K}$. In addition to these functions, to obtain $G(E, T)$ the model employes the software code in FORTRAN presented in [Mewe et al., 1986], where $G$ is considered as a function of wavelength $\lambda$ (in $\AA$ ) $(\lambda E=12.38)$ and temperature $T$ in $\mathrm{MK}$.

Formulas and sequence of computations

The basic formula for calculating spectral density has the form:

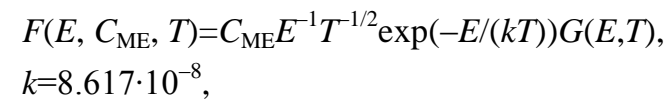

$$
E_{2}=E_{0}+\Delta E \text {. }
$$

For $\lambda_{i} \in\{1.4,4.6,6.1,9.1,14.2\}$ find the minimum value of $i$ satisfying the condition $12.38 / \lambda_{i} \leq E_{0}$.

If $i>1$ and $12.38 / \lambda_{i-1} \leq E_{2}$, then $E_{2}=E_{0}-\Delta E$.

Calculation

- short model:

$F_{1}=F_{\mathrm{XR}, \mathrm{XS}}\left(E_{1}\right), F_{2}=F_{\mathrm{XR}, \mathrm{XS}}\left(E_{2}\right)$,

$F_{0}=F_{1}$.

- complete model:

$F_{1}=F_{\mathrm{HXR}}\left(E_{1}\right), F_{2}=F_{\mathrm{HXR}}\left(E_{2}\right)$,

$F_{0}=F_{1} \exp \left(-\sigma\left(E_{0}\right) / \sigma\left(k T_{\mathrm{HXR}}\right)\right)$.

$A=\left(\ln \left(F_{2}\right)-\ln \left(F_{1}\right)\right) /\left(\ln \left(E_{2}\right)-\ln \left(E_{1}\right)\right) \quad(A<0)$,

$F_{\text {ext }}(E)=F_{0}\left(E / E_{0}\right)^{A}$.

Given that spectral parameters are independent of photon energy, they can be computed in a separate program block. Then, once obtained values can further be used to determine the desired spectral density at a given energy $E$. This approach significantly reduces the computation time.

\section{Appendix 2. Approximation of support functions of the HMSXS model}

The argument of desired function may be: $E$ - energy in $\mathrm{keV}, T$ - temperature in $\mathrm{K}, R$-dimensionless quantity.

For all the functions considered, we use the polynomial approximation $P_{N}(x)=\sum_{i=0}^{N} a_{i} x^{i}$. In all the cases, except for $K(T)$, polynomials approximate the decimal logarithm of desired function. For $K(T)$, we use the approximation of the function itself. The polynomial argument is the decimal logarithm of the argument of desired function. An exception is $T_{\mathrm{XL}} \mathrm{xS}(R)$ when $R<10$, where the polynomial argument $x=R$. Domains of definitions of the desired functions are divided into intervals in which polynomials are determined. Boundaries of the intervals are given by one of the following versions: argument of desired function, decimal logarithm of this argument; and for the functions of $T$, by $k T$ ( $k=8.617 \mathrm{e}-8 \mathrm{keV} / \mathrm{K}$ is the Boltzmann constant). Boundaries of the ranges, polynomial degrees and coefficients are listed in Tables A.1-A.10. Each table line represents one interval and lists the values in increasing order of the argument of desired function. The line shows the upper boundary of the range. Its lower boundary corresponds to the upper boundary of the previous range or to the lower boundary of the function domain. The Tables use the following notations: $E_{\mathrm{K}}(\mathrm{O})=0.53728 \mathrm{keV}, E_{\mathrm{K}}(\mathrm{Si})=1.8285$ $\mathrm{keV}, E_{\mathrm{K}}(\mathrm{Fe})=7.0834 \mathrm{keV}$.

Table A.1. Coefficients of $\sigma(E)$ approximation in the range $0.05-200 \mathrm{keV}$

\begin{tabular}{|c|c|c|c|c|c|c|}
\hline & $N$ & $a_{0}$ & $a_{1}$ & $a_{2}$ & $a_{3}$ & $a_{4}$ \\
\hline \multicolumn{7}{|c|}{$\lg \sigma=P_{N}(\lg E)$} \\
\hline$E<E_{\mathrm{K}}(\mathrm{O})$ & 1 & -22.014 & -2.677 & - & - & - \\
\hline$E<E_{\mathrm{K}}(\mathrm{Si})$ & 1 & -21.77 & -2.58 & - & - & - \\
\hline
\end{tabular}




\begin{tabular}{|c|c|c|c|c|c|c|}
\hline$E<E_{\mathrm{K}}(\mathrm{Fe})$ & 2 & -21.58 & -2.843 & 0.346 & - & - \\
\hline$E \leq 200$ & 4 & -17.59 & -13.54 & 10.493 & -3.614 & 0.459 \\
\hline
\end{tabular}

Table A.2. Coefficients of $K(T)$ approximation (0.4-0.05 nm XS)

\begin{tabular}{|c|c|c|c|c|c|}
\hline & $N$ & $a_{0}$ & $a_{1}$ & $a_{2}$ & $a_{3}$ \\
\hline & \multicolumn{5}{|c|}{$K=P_{N}(\lg T)$} \\
\hline$k T<E_{\mathrm{K}}(\mathrm{O})$ & 0 & 1 & - & - & - \\
\hline $\lg T<7.2$ & 3 & 199.062 & -87.5525 & 12.9103 & -0.635077 \\
\hline$k T<E_{\mathrm{K}}(\mathrm{Si})$ & 2 & -53.8482 & 15.6238 & -1.11363 & - \\
\hline $\lg T<7.6$ & 3 & -301.849 & 114.822 & -14.3516 & 0.58954 \\
\hline$k T<E_{\mathrm{K}}(\mathrm{Fe})$ & 3 & -540.196 & 213.24 & -27.8684 & 1.20712 \\
\hline $\lg T<8.1$ & 2 & 120.048 & -28.9866 & 1.75287 & - \\
\hline $\lg T<8.3$ & 3 & 998.077 & -356.617 & 42.508 & -1.69006 \\
\hline $\lg T<8.5$ & 3 & 620.494 & -218.296 & 25.6186 & -1.00269 \\
\hline $\lg T \leq 9.0$ & 3 & 85.1592 & -29.0526 & 3.31511 & -0.126334 \\
\hline
\end{tabular}

Table A.3. Coefficients of $K(T)$ approximation (0.3-0.05 nm XS)

\begin{tabular}{|c|c|c|c|c|c|}
\hline & $N$ & $a_{0}$ & $a_{1}$ & $a_{2}$ & $a_{3}$ \\
\hline & \multicolumn{5}{|c|}{$K=P_{N}(\lg T)$} \\
\hline$k T<E_{\mathrm{K}}(\mathrm{O})$ & 0 & 1 & - & - & - \\
\hline $\lg T<7.2$ & 3 & 146.34 & -63.9588 & 9.3879 & -0.45963 \\
\hline$k T<E_{\mathrm{K}}(\mathrm{Si})$ & 2 & -40.7695 & 11.8416 & -0.83991 & - \\
\hline $\lg T<7.7$ & 3 & 101.314 & -48.4152 & 7.6527 & -0.397818 \\
\hline$k T<E_{\mathrm{K}}(\mathrm{Fe})$ & 2 & 1.5471 & 1.1305 & -0.162286 & - \\
\hline $\lg T<8.1$ & 2 & 134.603 & -32.4009 & 1.95317 & - \\
\hline $\lg T<8.3$ & 3 & 1032.76 & -367.285 & 43.5751 & -1.72439 \\
\hline $\lg T<8.5$ & 3 & 650.159 & -228.143 & 26.7066 & -1.04268 \\
\hline $\lg T \leq 9.0$ & 3 & 100.152 & -34.0886 & 3.88033 & -0.147517 \\
\hline
\end{tabular}

Table A.4. Coefficients of $T_{\mathrm{XL}, \mathrm{XS}}(R)$ approximation (0.4-0.05 nm XS)

\begin{tabular}{|c|c|c|c|c|c|}
\hline & $N$ & $a_{0}$ & $a_{1}$ & $a_{2}$ & $a_{3}$ \\
\hline & \multicolumn{5}{|c|}{$\lg T=P_{N}(R)$} \\
\hline $0.72 \leq R<0.85$ & 3 & 41.56 & -115.3 & 135.520 & -53.99 \\
\hline$R<1.3$ & 3 & 13.045 & -10.9 & 8.1 & -2.16 \\
\hline$R<3$ & 3 & 9.3 & -1.72 & 0.521 & -0.0586 \\
\hline$R<7$ & 3 & 7.9745 & -0.3695 & 0.0496 & $-2.484 \mathrm{e}-3$ \\
\hline$R<10$ & 3 & 6.627 & 0.1774 & -0.02577 & $1.051 \mathrm{e}-3$ \\
\hline $\lg \lg T=P_{N}(\lg R)$ \\
\hline $\lg R<2.5$ & 3 & 7.454 & -0.7665 & 0.209 & -0.02282 \\
\hline $\lg R \leq 8.0$ & 3 & 6.996 & -0.258 & 0.0243 & $-9.706 \mathrm{e}-4$ \\
\hline
\end{tabular}

Table A.5. Coefficients of $T_{\mathrm{XL}, \mathrm{XS}}(R)$ approximation $(0.3-0.05 \mathrm{~nm}$ XS)

\begin{tabular}{|c|c|c|c|c|c|}
\hline & $N$ & $a_{0}$ & $a_{1}$ & $a_{2}$ & $a_{3}$ \\
\hline & \multicolumn{5}{|c|}{$\lg T=P_{N}(R)$} \\
\hline $0.72 \leq R<1$ & 3 & 34.6 & -82.12 & 87.16 & -31.4 \\
\hline$R<1.9$ & 3 & 10.39 & -3.563 & 1.71 & -0.3017 \\
\hline$R<4$ & 3 & 8.705 & -0.79 & 0.1697 & -0.0137 \\
\hline$R<10$ & 3 & 7.95 & -0.214 & 0.0211 & $-7.89 \mathrm{e}-4$ \\
\hline \multicolumn{7}{|c|}{$\lg T=P_{N}(\lg R)$} \\
\hline $\lg R<3.3$ & 3 & 7.754 & -0.8157 & 0.2172 & -0.02345 \\
\hline $\lg R \leq 8.0$ & 3 & 7.09 & -0.197 & 0.0149 & $-5 \mathrm{e}-4$ \\
\hline
\end{tabular}


Table A.6. Coefficients of $T^{*}{ }_{\mathrm{QD}, \mathrm{XL}}(R)$ approximation

\begin{tabular}{|c|c|c|c|c|c|}
\hline & $N$ & $a_{0}$ & $a_{1}$ & $a_{2}$ & $a_{3}$ \\
\hline & \multicolumn{5}{|c|}{$\lg T=P_{N}(\lg R)$} \\
\hline $0.64 \leq \lg R<1.7475$ & 3 & 7.116 & -0.655 & 0.2487 & -0.045 \\
\hline $\lg R<5.0$ & 3 & 6.9035 & -0.3096 & 0.0420 & -0.00255 \\
\hline $\lg R \leq 7.95$ & 2 & 6.5849 & -0.123 & 0.0047 & - \\
\hline
\end{tabular}

Table A.7. Coefficients of $R_{\mathrm{QD}, \mathrm{XL}}(T)$ approximation

\begin{tabular}{|c|c|c|c|c|c|}
\hline & $N$ & $a_{0}$ & $a_{1}$ & $a_{2}$ & $a_{3}$ \\
\hline & \multicolumn{5}{|c|}{$\lg R=P_{N}(\lg T)$} \\
\hline $6.5 \mathrm{e} 5 \leq T<3 \mathrm{e} 6$ & 3 & 3342.13 & -1520.14 & 231.342 & -11.7747 \\
\hline $\lg T<7.1$ & 3 & 888.463 & -369.335 & 51.3451 & -2.38583 \\
\hline $\lg T<8.0$ & 3 & 121.656 & -43.981 & 5.31888 & -0.21498 \\
\hline $\lg T \leq 9.0$ & 3 & 38.7631 & -13.0515 & 1.47102 & -0.05537 \\
\hline
\end{tabular}

Table A.8. Coefficients of $R_{\mathrm{QD}, \mathrm{XL}}^{*}(T)$ approximation

\begin{tabular}{|c|c|c|c|c|c|}
\hline & $N$ & $a_{0}$ & $a_{1}$ & $a_{2}$ & $a_{3}$ \\
\hline \multicolumn{7}{|c|}{$\lg R=P_{N}(\lg T)$} \\
\hline $6 . \leq \lg T<6.2$ & 2 & 609.025 & -185.646 & 14.1969 & - \\
\hline$T<3 \mathrm{e} 6$ & 3 & 3551.05 & -1617.25 & 246.431 & -12.559 \\
\hline$k T<E_{\mathrm{K}}(\mathrm{O})$ & 2 & 228.258 & -64.708 & 4.5928 & - \\
\hline $\lg T \leq 7.32$ & 2 & 116.47 & -31.678 & 2.1546 & - \\
\hline $\lg T \leq 9.0$ & 0 & 0 & - & - & - \\
\hline
\end{tabular}

Table A.9. Coefficients of $J_{\mathrm{XL}}(T)$ approximation

\begin{tabular}{|c|c|c|c|c|c|}
\hline & $N$ & $a_{0}$ & $a_{1}$ & $a_{2}$ & $a_{3}$ \\
\hline & \multicolumn{5}{|c|}{$\lg J=P_{N}(\lg T)$} \\
\hline $5.6 \leq \lg T<6$ & 3 & -5201.24 & 2422.86 & -378.004 & 19.73 \\
\hline $\lg T<6.1$ & 2 & -824.12 & 253.513 & -19.652 & - \\
\hline$T<3 \mathrm{e} 6$ & 3 & -2868.62 & 1277.343 & -190.524 & 9.5042 \\
\hline $\lg T<7.2$ & 3 & -498.87 & 198.792 & -26.773 & 1.21014 \\
\hline $\lg T \leq 9.0$ & 3 & -116.82 & 38.077 & -4.2348 & 0.15645 \\
\hline
\end{tabular}

Table A.10. Coefficients of $J^{*}{ }_{\mathrm{XL}}(T)$ approximation

\begin{tabular}{|c|c|c|c|c|c|}
\hline & $N$ & $a_{0}$ & $a_{1}$ & $a_{2}$ & $a_{3}$ \\
\hline & \multicolumn{5}{|c|}{$\lg J=P_{N}(\lg T)$} \\
\hline $5.77 \leq \lg T<6$ & 3 & -6087.54 & 2881.33 & -456.986 & 24.2617 \\
\hline$T<3 \mathrm{e} 6$ & 3 & -2956. & 1319.33 & -197.247 & 9.8629 \\
\hline$k T<E_{\mathrm{K}}(\mathrm{O})$ & 3 & -912.763 & 382.865 & -54.0406 & 2.5555 \\
\hline$k T<E_{\mathrm{K}}(\mathrm{Si})$ & 3 & -207.381 & 68.845 & -7.458 & 0.25287 \\
\hline$k T<E_{\mathrm{K}}(\mathrm{Fe})$ & 3 & 1105.34 & -448.034 & 60.3084 & -2.70509 \\
\hline $\lg T<8.4$ & 3 & -1165.44 & 434.003 & -53.8797 & 2.22234 \\
\hline $\lg T \leq 9.0$ & 3 & 799.748 & -275.972 & 31.617 & -1.20947 \\
\hline
\end{tabular}

